\title{
High mobility group box 1-mediated autophagy promotes neuroblastoma cell chemoresistance
}

\author{
LI WANG, HUIPING ZHANG, MIN SUN, ZHANGHUA YIN and JIHONG QIAN \\ Department of Neonatology, Xinhua Hospital, Shanghai Jiaotong University School of Medicine, \\ Shanghai 200092, P.R. China
}

Received May 25, 2015; Accepted July 27, 2015

DOI: $10.3892 /$ or.2015.4278

\begin{abstract}
Neuroblastoma (NB) is one of the most common tumors in childhood. Unfortunately, the survival outcomes remain unsatisfactory since NB commonly develops multidrug resistance. Recent studies have demonstrated that the high mobility group box 1 (HMGB1)-mediated autophagy promotes chemoresistance in osteosarcoma, lung adenocarcinoma and ovarian cancer, but the exact molecular mechanism underlying HMGB1-mediated autophagy in NB has not been clearly defined. In the present study, we investigated the role of HMGB1 in the development of resistance to anticancer agents in NB. Anticancer agents including doxorubicin, cisplatin and etoposide each induced HMGB1 upregulation, promoted cytosolic HMGB1 translocation and the elevation of autophagic activity in human NB cells. RNA interference-mediated knockdown of HMGB1 restored the chemosensitivity of NB cells. Furthermore, mechanistic investigation revealed that HMGB1 promoted the proliferative activity and invasive potential of NB cells. HMGB1 enhanced drug resistance by inducing Beclin-1-mediated autophagy, an intracellular self-defense mechanism known to confer drug resistance. In addition, we found that HMGB1 facilitated autophagic progression and reduced oxidative stress induced by doxorubicin. Therefore, through its role as a regulator of autophagy, HMGB1 is a critical factor in the development of chemoresistance and tumorigenesis, and it may be a novel target for improving the efficacy of NB therapy.
\end{abstract}

\section{Introduction}

Neuroblastoma (NB), one of the most common tumors in childhood, accounts for $\sim 10 \%$ of all pediatric cancers and $15 \%$ of childhood cancer-related mortality (1). The survival

Correspondence to: Dr Jihong Qian, Department of Neonatology, Xinhua Hospital, Shanghai Jiaotong University School of Medicine, 1665 Kongjiang Road, Shanghai 200092, P.R. China

E-mail: qianjh668@163.com

Key words: HMGB1, autophagy, Beclin-1, proliferation, invasion, chemoresistance outcomes of NB remain unsatisfactory, although a variety of surgical techniques have been developed in the past few decades. One of the clinical hallmarks of NB is multidrug resistance (2), and many patients, particularly those with malignant NB, often develop chemoresistance (3). It is well established that tumor cells are able to resist chemotherapeutic agents through a variety of mechanisms, which includes enhancing drug metabolism, altering the accumulation of medicine.

High mobility group box 1 (HMGB1), a conserved nonhistone nuclear protein, binds DNA and promotes the assembly of proteins with a specific DNA target site (4). In addition to its role in transcription, HMGB1 also functions in the cytoplasm as an extracellular signaling protein during tumor progression. HMGB1 is closely associated with each of the hallmarks of cancer, including cell proliferation, ability to develop angiogenesis, evasion of apoptosis, tissue invasion and metastasis and represents a potential target in the therapy of various types of cancers $(5,6)$. It has been reported that HMGB1 is highly expressed in hepatocellular carcinoma (7), colorectal cancer (8), lymphoma (9) and breast cancer (10). Increased expression of HMGB1 is correlated with progression and poor prognosis in human nasopharyngeal carcinoma (11) and colorectal cancer (12). In addition, targeting HMGB1 by RNA interference was found to inhibit ovarian cancer growth and metastasis in vitro (13).

It has been widely reported that HMGB1 plays a role in facilitating autophagy $(14,15)$, since it can disrupt the interaction between Beclin-1 and its negative regulator Bcl-2 by competitively binding to Beclin-1 (16). Moreover, recent studies suggest that HMGB1-mediated autophagy promotes chemoresistance in osteosarcoma, lung adenocarcinoma and ovarian cancer $(4,17,18)$. These studies found that the level of HMGB1 protein increased after anticancer agent treatment, and HMGB1 protein contributed to inducing autophagy to evade apoptosis. Therefore, HMGB1 is a newly identified gene associated with cancer growth and metastasis, as well as tumor chemoresistance. Moreover, HMGB1-mediated autophagy is considered as a potential marker of therapeutic effect and may be used to predict clinical outcome. However, the mechanism and significance of HMGB1-mediated autophagy in NB remain largely unknown.

In the present study, we found that HMGB1 expression levels, particularly in the cytoplasm, increased rapidly in response to anticancer agents including doxorubicin (Dox), cisplatin (Cis) 
and etoposide (Eto). RNA interference-mediated knockdown of HMGB1 restored the chemosensitivity of SH-SY5Y cells. Overexpression of HMGB1 promoted cell growth and migration in vitro and increased chemotherapy resistance. Furthermore, the results revealed that HMGB1-overexpressing SH-SY5Y cells acquired resistance to multidrug treatment through regulation of autophagy, an intracellular self-defense mechanism known to confer drug resistance. We found that HMGB1 promoted cell growth and migration in vitro and increased chemotherapy resistance by facilitating autophagic progression. Therefore, HMGB1 is a critical factor in the development of chemoresistance and it offers a novel target for improving the efficacy of NB therapy.

\section{Materials and methods}

Cell culture and regents. The NB SH-SY5Y cell line used in the experiments was purchased from the Institute of Biochemistry and Cell Biology (Shanghai, China). The cells were cultured in Dulbecco's modified Eagle's medium (DMEM) (Gibco, Life Technologies) supplemented with $10 \%$ fetal bovine serum (FBS; Gibco, Uruguay), $100 \mathrm{U} / \mathrm{ml}$ of penicillin and $100 \mu \mathrm{g} / \mathrm{ml}$ of streptomycin. They were cultured in a humidified atmosphere containing $5 \% \mathrm{CO}_{2}$ at $37^{\circ} \mathrm{C}$. Lipofectamine 2000 was purchased from Invitrogen (Carlsbad, CA, USA). 7'-Dichlorofluorescein diacetate (DCFH-DA), Cell Counting Kit-8 (CCK-8) and the ECL-Plus kit were purchased from Beyotime (China); M-MLV reverse transcriptase was purchased from Promega (Madison, WI, USA); all antibodies were purchased from Santa Cruz Biotechnology (Santa Cruz, CA, USA). 3-Methyladenine (3-MA) was purchased from Sigma (St. Louis, MO, USA).

GFP-LC3 transfection. The green fluorescent proteinLC3 (GFP-LC3) plasmid was purchased from GeneChem (China). Cells were seeded at a density of $9 \times 10^{5} / \mathrm{ml}$ on glass coverslips placed into 24-well tissue culture plates (Corning Glass Works, Corning, NY, USA). On the following day, the cells were transfected at 50-80\% confluence and transfected with the GFP-LC3 plasmid according to the manufacturer's instructions. After $4 \mathrm{~h}$, the medium was replaced by DMEM containing 10\% FBS, and the cells were left for another $24 \mathrm{~h}$. The stable cells were selected according to our previous study (19).

CCK-8 assay for cell proliferation. Cells were plated in 96-well culture plates $\left(5 \times 10^{3}\right.$ cells/well). At the indicated time, viable cell numbers were determined by a cell proliferation assay using CCK-8. The absorbance of optical densities at each time point was detected by a microplate spectrophotometer at $450 \mathrm{~nm}$.

Overexpression of HMGB1. The HMGB1 gene was amplified by polymerase chain reaction (PCR) and was inserted into the Agel site of the pGC-FU-3FLAG vector plasmid (Addgene, Cambridge, MA, USA). For stable overexpression of HMGB1, HEK293T cells were plated in $75 \mathrm{~cm}^{2}$ culture flasks and transfected with $10 \mu \mathrm{g}$ lentivirus-HMGB1 vector (lenti-HMGB1) or lentivirus-GFP vectors (lenti-GFP). The medium was changed the next day, and the viral supernatant was harvested
$48 \mathrm{~h}$ later. All medium containing viruses were collected and passed through $0.45-\mu \mathrm{m}$ syringe filters. SH-SY5Y cells were incubated with the lentivirus supernatant for $24 \mathrm{~h}$ and selected with $2 \mu \mathrm{g} / \mathrm{ml}$ puromycin (Sigma) according to the manufacturer's instructions.

Knockdown of HMGB1, Beclin-1 and Atg5. Transfection with HMGB1-siRNA, Beclin-1-siRNA and Atg5-siRNA (Sigma) was carried out by the Lipofectamine 2000 transfection reagent according to the manufacturer's instructions.

Analysis of apoptosis by flow cytometry. The incidence of apoptosis in NB cells was detected using the Annexin V-FITC/PI apoptosis detection kit (BD Pharmingen, USA) as previously described (20). Apoptotic cells, including those staining positively for Annexin V-FITC and negatively for PI and those that were double-positive, were counted and represented as a percentage of the total cell count.

Western blotting assay. The cells were extracted at the indicated time using lysis buffer. Protease and phosphatase with whole-cell extracts were prepared in RIPA buffer. Cell extracts were boiled for $10 \mathrm{~min}$ in loading buffer and then equal amounts of cell extracts were separated on 6-15\% SDS-PAGE gels. Separated protein bands were transferred to polyvinylidene fluoride (PVDF) membranes, and the membranes were blocked in 5\% skim milk powder. The primary antibodies against HMGB1, Beclin-1, Atg5, cyclin E, cyclin B1 and $\beta$-actin were diluted according to the instructions concerning the antibodies and incubated overnight at $4^{\circ} \mathrm{C}$. Subsequently, horseradish peroxidase-linked secondary antibodies were incubated at room temperature for $4 \mathrm{~h}$ at a dilution ratio of 1:1,000 according to the kit's instructions. The membranes were washed with TBST for three times ( 5 min each time), and the immunoreactive bands were visualized using the ECL-Plus kit. The relative protein level was normalized to $\beta$-actin concentration.

Autophagolysosome detection by transmission electron microscopy. At the indicated time, the cells were fixed in $0.2 \%$ glutaraldehyde in PBS ( $\mathrm{pH}$ 7.4) for $2 \mathrm{~h}$ at room temperature, post-fixed in $1 \%$ osmium tetroxide in water for $1 \mathrm{~h}$, and then stained in $2 \%$ uranyl acetate in water for $1 \mathrm{~h}$ in the dark. After dehydration in an ascending series of ethanol, the samples were embedded in Durcopan ACM for $6 \mathrm{~h}$, and cut into 80-nm sections. These sections were stained with uranyl acetate and lead citrate and examined with a transmission electron microscope (Philips CM, The Netherlands).

Measurements of intracellular ROS. To determine ROS generation within $\mathrm{H}_{2} \mathrm{O}_{2}$-treated cells, flow cytometry was performed. Cells were exposed to $\mathrm{H}_{2} \mathrm{O}_{2}$ for different hours and then stained with $5 \mu \mathrm{g} / \mathrm{ml}$ of DCFH-DA for $30 \mathrm{~min}$ and subjected to flow cytometry and analyzed by CellQuest software (Becton-Dickinson, San Jose, CA, USA) according to a previously described method (21).

Wound-healing assay. A wound-healing assay was also performed to confirm the influence of HMGB1 on SH-SY5Y cell migration. When the cells transfected with lenti-HMGB1 

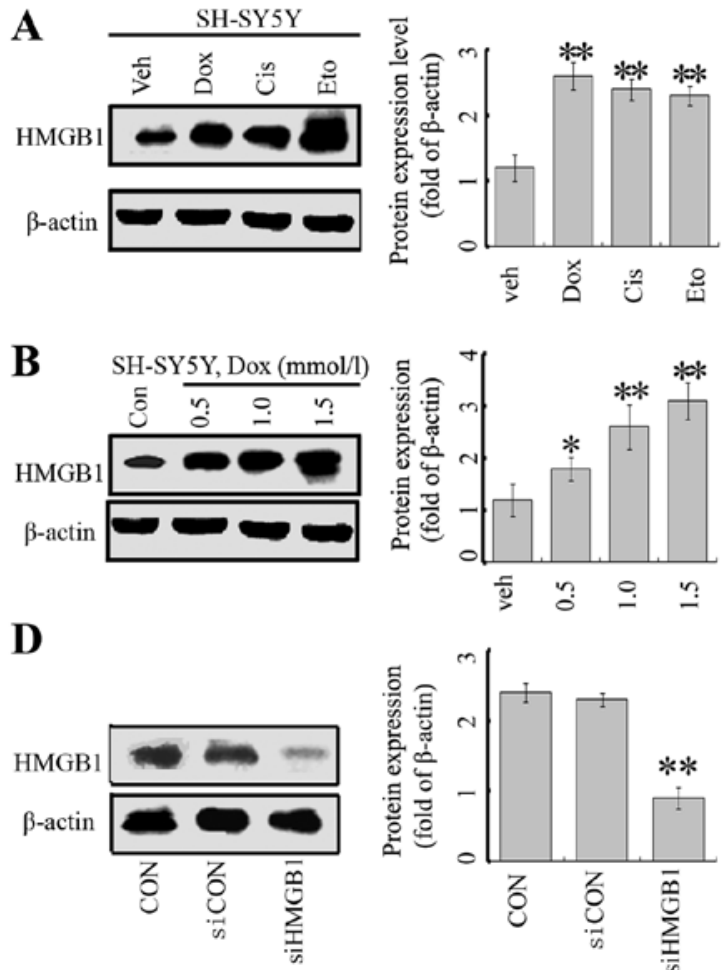
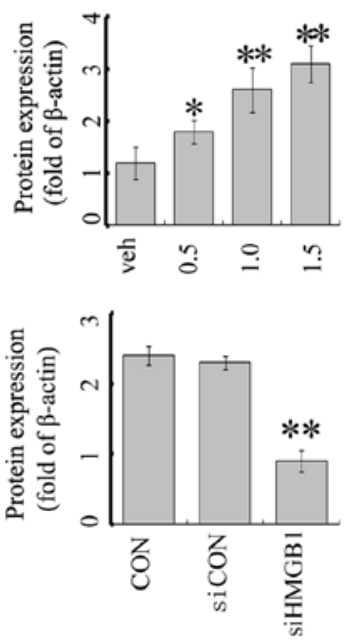
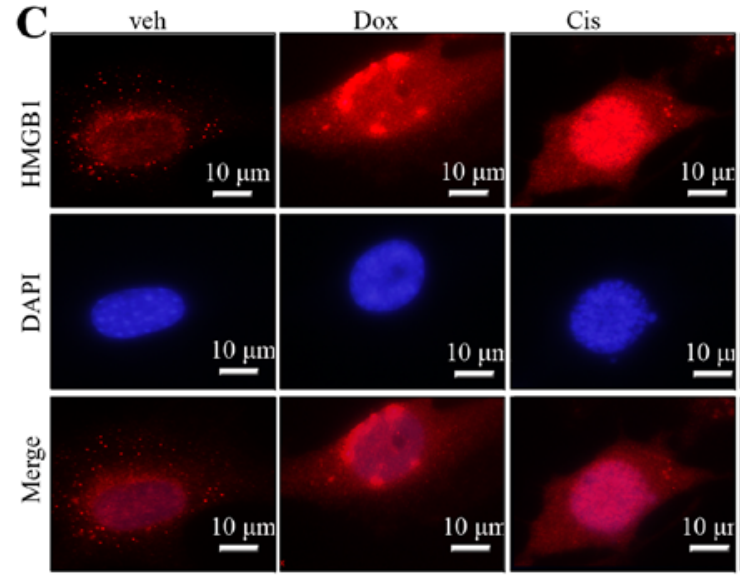

E

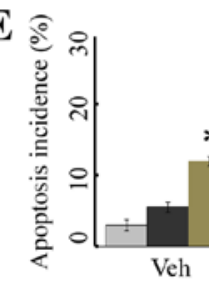

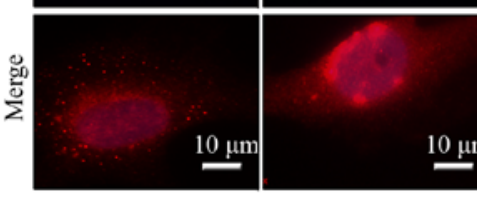

.

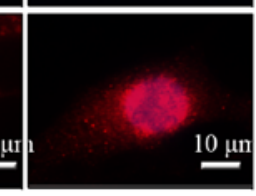

$10 \mu$

Figure 1. Anticancer agents induce HMGB1 expression in neuroblastoma cells. (A) SH-SY5Y cells were treated with Dox (1 $\mu \mathrm{M})$, Cis (20 $\mu \mathrm{M})$, Eto (10 $\mu \mathrm{M})$ or vehicle (distilled water) for $24 \mathrm{~h}$, and the HMGB1 protein level was analyzed by western blotting (WB) (vs. untreated group). (B) The cells were treated with Dox at concentrations of $0.5,1$ and $1.5 \mathrm{mmol} / 1$, respectively, and the HMGB1 level was tested by WB assays. (C) SY-SH5Y cells were treated with anticancer agents, and the HMGB1 protein was detected by IF assays. (D) SY-SH5Y cells were transfected with siCON or siHMGB1, and the level of HMGB1 protein was suppressed in the siHMGB1 group. The cells without transfection served as the control (CON). ${ }^{* *} \mathrm{P}<0.01$ vs. CON. (E) Flow cytometric analysis of apoptosis incidence. Cells in the siHMGB1, siCON and CON groups were treated with Dox $(1 \mu \mathrm{M})$, Cis $(20 \mu \mathrm{M})$, Eto $(10 \mu \mathrm{M})$ or vehicle (distilled water) for $24 \mathrm{~h}$, and the apoptosis was assessed by flow cytometry with Annexin V/FITC staining. The results are the representative of three identical experiments and the bars are the mean $\pm \mathrm{SD}$. ${ }^{*} \mathrm{P}<0.05$ vs. control, ${ }^{* *} \mathrm{P}<0.01$ vs. control.

or the lentivirus were grown to confluency, a scratch in the cell monolayer was made with a cell scratch spatula. After the cells were incubated under standard conditions for 24 and $48 \mathrm{~h}$, images of the scratches were captured using a digital camera system coupled with a microscope.

Transwell migration assays. The invasion assays were performed using 24-well Transwell chambers ( $8 \mu \mathrm{m}$; Corning). For the invasion assay, tumor cells were resuspended in serum-free DMEM, and $2 \times 10^{5}$ cells were seeded into the upper chambers. DMEM $(0.5 \mathrm{ml})$ containing $10 \%$ FBS was added to the bottom chambers. Following a 24-h incubation, cells on the upper surface of the membrane were scrubbed off, and the migrated cells were fixed with $75 \%$ ethanol, stained with $0.1 \%$ crystal violet and counted under a light microscope.

Statistical analysis. SPSS 13.0 was used for statistical analysis. One-way analysis of variance (ANOVA) was used to analyze the differences between groups. The LSD method of multiple comparisons was used when the probability for ANOVA was statistically significant. Statistical significance was set at $\mathrm{P}<0.05$

\section{Results}

Anticancer agents promote HMGBl expression, and knockdown of HMGBI increases sensitivity to chemotherapy in NB cells. First, we assayed the effects of the anticancer agents, Dox, Cis and Eto on the expression of HMGB1. These anticancer agents significantly enhanced the expression of HMGB1 in the SH-SY5Y cells (Fig. 1A). Moreover, this effect was dose-dependent in the case of Dox (Fig. 1B). In addition, immunofluorescence indicated that in the absence of treatment, HMGB1 was mainly located in the nucleus, with very low levels in the cytoplasm. Treatment of anticancer agents markedly enhanced total levels of HMGB1 in both the nucleus and cytoplasm (Fig. 1C).

To explore the potential role of HMGB1 in the regulation of cell death in NB cells, a target-specific siRNA against HMGB1 (siHMGB1) was transfected into SH-SY5Y cells. The transfection inhibited HMGB1 expression, evidenced by a decrease in the HMGB1 protein level (Fig. 1D). The inhibition of HMGB1 rendered cells more sensitive to Dox-, Cis- and Eto-induced cell injury, by assessment of the incidence of apoptosis (Fig. 1E).

Effect of HMGB1 on NB cell proliferation, invasion, metastasis and cell cycle distribution. To further explore the function of HMGB1 and its mechanism, the HMGB1 gene was overexpressed by a lentivirus in the SH-SY5Y cells. A lentivirus vector containing the HMGB1 gene (lenti-HMGB1) was transfected into SH-SY5Y cells, and the infection efficiency was $>90 \%$ as detected by fluorescence microscopy (Fig. 2A). As expected, the HMGB1 expression was markedly enhanced 

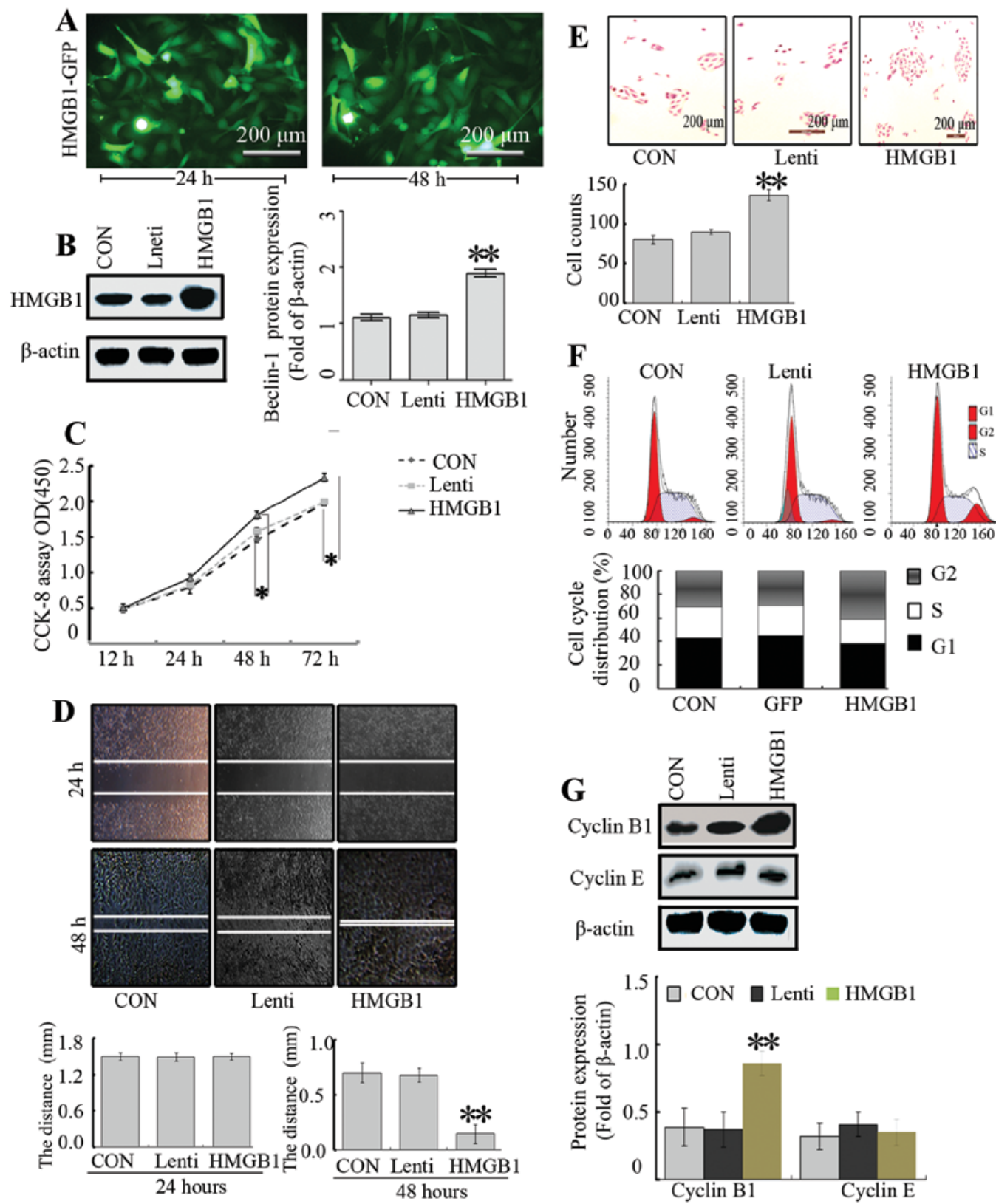

Figure 2. Effect of HMGB1 on neuroblastoma cell proliferation, invasion, metastasis and cell cycle distribution. (A) Fluorescence microscopy for lenti-HMGB1. The lentivirus vector containing HMGB1 was constructed and transfected into SH-SY5Y cells. The infection efficiency (at an MOI of 100 ) was $>90 \%$ at $24 \mathrm{~h}$ (B) Western blotting assay for the expression of HMGB1. SY-SH5Y cells were transfected with lentivirus vector containing the HMGB1 gene (HMGB1 group) or without (Lenti), and the cells without transfection served as the control (CON). The HMGB1 level in the HMGB1-overexpressing cells was increased significantly. (C) CCK-8 assay for cell growth. The growth of SH-SY5Y cells was detected at 12, 24, 48 and $72 \mathrm{~h}$ after transfection. The cell viability was increased in the HMGB1 group. (D) The wound-scratch assay of HMGB1-overexpressing cells. The migratory capacity of cells in the HMGB1 group was markedly higher than that in the CON and Lenti groups. (E) Transwell invasion assay to assess the transmembrane invasive ability. The quantitative results showed an increased ability in the HMGB1-overexpressing cells, compared with the CON and Lenti groups. (F) Cell cycle distribution was analyzed by flow cytometric analysis. The percentage of cells in each phase of the cell cycle showed that HMGB1 reduced cells in G0/G1 and increased cells in the S phase. (G) Western blotting assay for the expression of cyclin B1 and cyclin E. The level of cyclin B1 protein was lower in the HMGB1 group, compared with the levels in the CON. ${ }^{*} \mathrm{P}<0.05$ vs. $\mathrm{CON},{ }^{* *} \mathrm{P}<0.01$ vs. CON.

after transfection at $48 \mathrm{~h}$ (Fig. 2B). In order to test the effect of HMGB1 overexpression on cell growth, we investigated the proliferative activity of the cells by CCK-8. As shown in Fig. 2C, overexpression of HMGB1 intensified the SH-SY5Y cell growth, compared with the growth noted in the Lenti and CON groups.

To determine the effect of HMGB1 on NB cell invasion and migration, Transwell and wound-healing assays were carried out. The migratory ability of the cells in the HMGB1 group was markedly higher when compared with that in the CON and Lenti groups (Fig. 2D). However, there were no significant differences between the Lenti and CON groups. Furthermore, a Transwell assay was performed to determine the ability of cells to invade a matrix barrier, and the representative micrographs of Transwell filters are presented in Fig. 2E. The invasive cell count demonstrated that invasive potential was 
$\mathbf{A}$
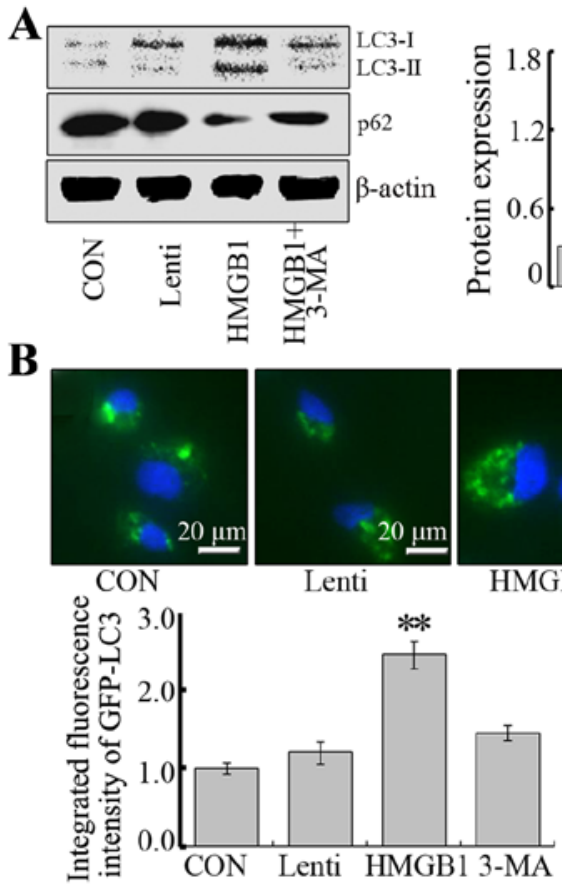

C

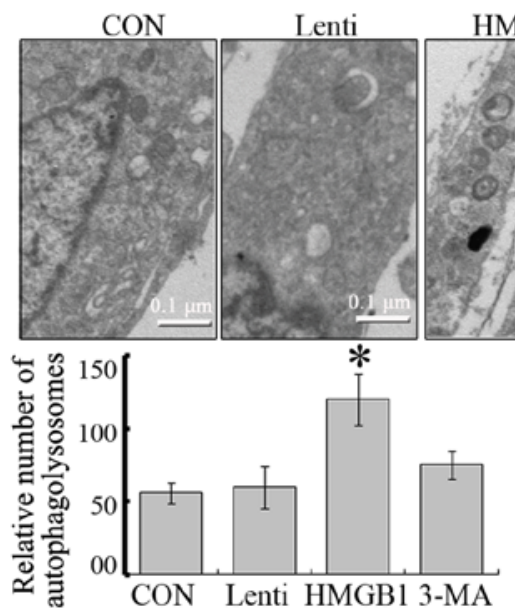

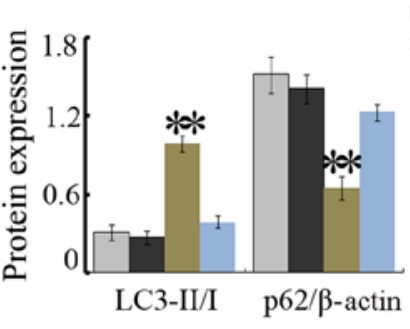

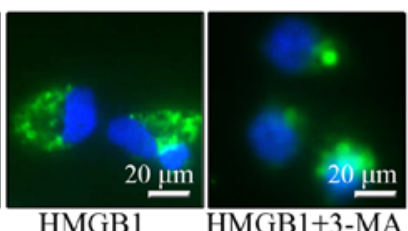

HMGB1+3-MA

\section{$\mathbf{E}$}
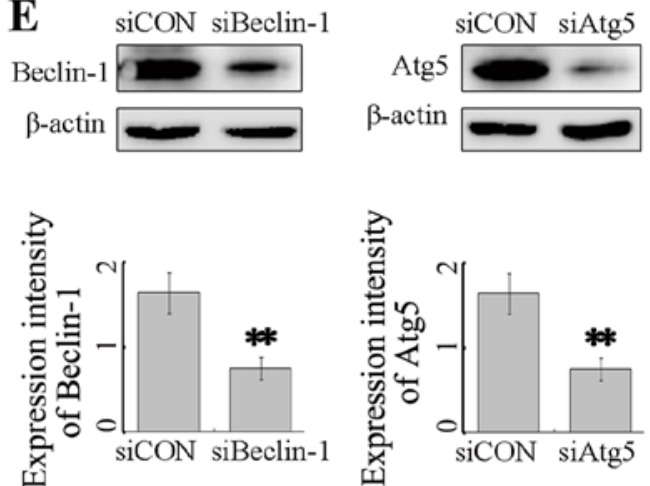

F

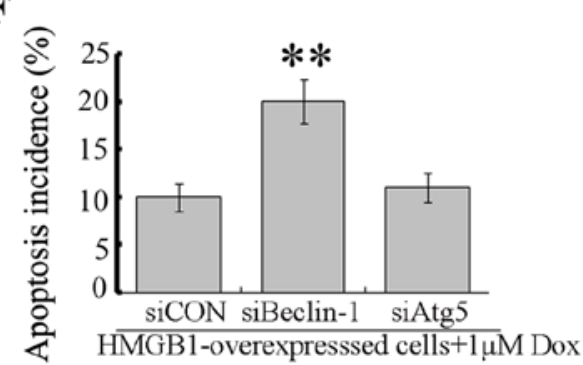

Figure 3. HMGB1-mediated autophagy alters the sensitivity of neuroblastoma cells to anticancer agents. (A) Western blot assay of the expression of LC3-I, LC3-II and p62. SY-SH5Y cells were transfected with the HMGB1-lentivirus vector and were treated with $5 \mathrm{mM} 3$-MA or vehicle for $24 \mathrm{~h}$. The conversion rate of LC3-I to LC3-II proteins increased and the p62 protein level was decreased in the HMGB1 group. 3-MA partly reversed the effect of HMGB1-mediated autophagy; ** P<0.01 vs. CON. (B) Immunofluorescence assay for GFP-LC3. The cells described in A were co-transfected with GFP-LC 3 and then observed by immunofluorescence microscope. More GFP-LC3-labeled vacuoles were accumulated in the cytoplasm of the HMGB1-overexpressing cells and 3-MA partly reversed the effect of HMGB1; ${ }^{* *} \mathrm{P}<0.01$ vs. CON. (C) Transmission electron microscopic assay of autophagosome formation. The cells described in A were detected by transmission electron microscopy. Consistently, the result was similar to that of B; ${ }^{*} \mathrm{P}<0.05 \mathrm{vs}$. CON. (D) Apoptosis was analyzed by flow cytometry (Annexin V-FITC/PI). Both cells without transfection (CON) and HMGB1-overexpressing cells were treated with Dox $(1 \mu \mathrm{M})$ for $24 \mathrm{~h}$ in combination with 3-MA or vehicle. Dox treatment led to an increase in apoptosis incidence and HMGB1-overexpressing cells were less sensitive to Dox treatment, while 3-MA partly reversed the effect of HMGB1; "P<0.01 vs. cells treated with Dox. (E) HMGB1-overexpressing cells were co-transfected with Beclin-1 siRNA (siBeclin-1) or Atg5 siRNA (siAtg5). The expression levels of Beclin-1 and Atg5 proteins were significantly reduced in the siBeclin-1 and siAtg5 group, respectively; ${ }^{*} \mathrm{P}<0.05$ vs. siCON. (F) Flow cytometry (Annexin V-FITC/PI) of apoptosis. The cells described in E were treated with Dox (1 $\mu \mathrm{M})$ for $24 \mathrm{~h}$ and then apoptosis incidence was analyzed. Knockdown of Beclin-1 rendered HMGB1-overexpressing cells more sensitive to Dox treatment, yet knockdown of Atg5 failed to increase the apoptosis induced by Dox treatment; ${ }^{\mathrm{P}}<0.05$ vs. siCON.

significantly increased in the HMGB1 group relative to the CON and Lenti groups.

Next, to understand the effects of HMGB1 on the cell cycle distribution of SH-SY5Y NB cells, flow cytometry was carried out. Flow cytometry revealed that the proportion of cells in the $\mathrm{G} 2 / \mathrm{M}$ phase in the HMGB1 group was higher than that in the CON and Lenti groups, while the percentage of cells in the $\mathrm{S}$ phase was not altered $(\mathrm{P}<0.01$, Fig. $2 \mathrm{~F})$. Subsequently, cyclin $\mathrm{B} 1$ and cyclin $\mathrm{E}$ are responsible for cell cycle progres- sion in the G2 phase (22) and thus were assessed by western blotting. The results showed that overexpression of HMGB1 led to an increased level of cyclin B1, but no significant changes in the levels of cyclin E were noted (Fig. 2G), suggesting that HMGB1 modulates the cell cycle through regulation of cyclin B1.

HMGBI activates autophagy and reduces the sensitivity to anticancer agents in vitro. Previous studies have reported that 

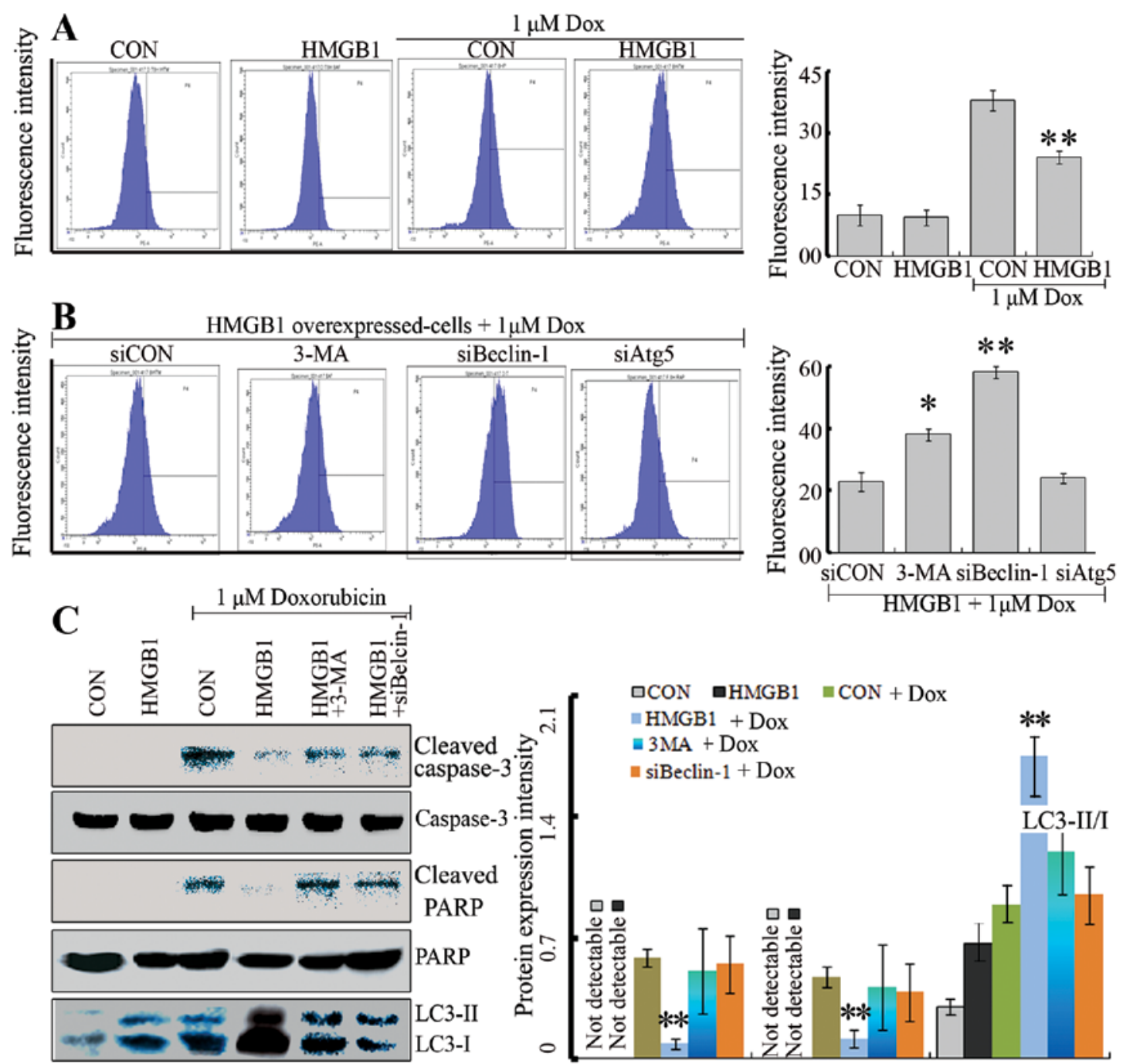

Cleaved caspase-3/caspase-3 Cleaved PARP/PARP LC3-II/I

Figure 4. HMGB1 decreases Dox-induced oxidative stress and cell death. (A) Flow cytometric assay of Dox-induced ROS in SY-SH5Y cells transfected with HMGB1 or without. SY-SH5Y cells (CON) and HMGB1-overexpressing cells were treated with Dox $(1 \mu \mathrm{M})$ or vehicle for 24 h. Subsequently, the ROS level was detected by flow cytometry. HMGB1 did not alter the basal ROS level while it reduced the increased ROS level induced by Dox; ${ }^{* *} \mathrm{P}<0.05$ vs. cells treated with cells. (B) Flow cytometry for Dox-induced ROS in HMGB1-overexpressing cells following inhibition of autophagy by pharmacological inhibitor (3-MA) and gene interference (siBelcin-1 or siAtg5). The HMGB1-overexpressing cells were treated with $5 \mathrm{mM}$ 3-MA, co-transfected with siBeclin-1 or siAtg5, and were then treated with Dox $(1 \mu \mathrm{M})$ for $24 \mathrm{~h}$. Dox-induced ROS in HMGB1-overexpressing cells was promoted by siBeclin-1 or 3-MA, rather than Atg5; ${ }^{* *} \mathrm{P}<0.05$ vs. siCON. (C) Dox treatment induced expression of cell death markers, and HMGB1-mediated autophagy reduced the cell death. Cells as described in A and B were treated with Dox $(1 \mu \mathrm{M})$ or vehicle for $24 \mathrm{~h}$ and then cleaved PARP, PARP, cleaved caspase-3, caspase-3 and LC3-II/I protein levels were assessed by western blotting. The cleaved PARP, cleaved caspase-3 and LC3-II expression levels were normalized against PARP, caspase-3 and LC3-I, respectively. Dox treatment led to an increase in cleaved PARP and cleaved caspase-3. HMGB1 partly reversed the increase in cleaved PARP and cleaved caspase-3 induced by Dox treatment. However, the protective effect of HMGB1 against Dox was reversed when autophagy was inhibited by pharmacological inhibitor (3-MA) ors gene interference (siBeclin-1); ** $\mathrm{P}<0.05$ vs. CON cells treated with Dox.

HMGB1-mediated autophagy is a significant contributor to chemotherapy resistance in several types of malignant tumors $(4,17,18)$. To investigate whether HMGB1 is a direct activator of autophagy that protects SH-SY5Y cells from apoptosis, we further evaluated the LC3-I to LC3-II conversion, p62 and GFP-LC3 puncta by fluorescent analysis. Overexpression of HMGB1 increased the appearance of LC3-II and the degradation of p62, promoting the formation by GFP-LC3 (Fig. 3A and B). However, this elevated flux of autophagy was abolished with 3-MA, an autophagy inhibitor (Fig. 3A). Consistently, fluorescence micrographs showed that the puncta of GFP-LC3 in HMGB1-overexpressing NB cells could be partly attenuated by 3-MA (Fig. 3A). Moreover, ultrastructure analysis revealed that HMGB1-overexpressing cells exhibited more autophagosomes compared with this number in the control cells in the NB cells, and 3-MA reduced the effect of HMGB1 (Fig. 3C). These data suggest that HMGB1 is a positive regulator of autophagy in NB cells.

To clarify the role of HMGB1-mediated autophagy in NB cells following chemotherapy, we analyzed the responses of HMGB1-overexpressing NB cells to Dox treatment. Dox induced apoptotic cell death to a great extent while overexpression of HMGB1 partly reversed the effect of Dox (Fig. 3D). Next, to investigate the role of HMGB1-mediated autophagy, autophagy was suppressed by 3-MA, an autophagy inhibitor by inhibiting class III PI3K (23), and RNA interfering technique 
targeted at Beclin-1 or Atg5 (Fig. 3E). As a result, treatment with 3-MA or Beclin-1 siRNA reversed HMGB1-induced protection against Dox. Nevertheless, blockade of autophagy by Atg 5 siRNA failed to reverse HMGB1-induced protection (Fig. 3F).

HMGB1 decreases Dox-induced oxidative stress and celldeath. Previous studies have demonstrated that Dox treatment leads to an increase in intracellular reactive oxygen species (ROS), oxidative stress injury, and cell death (24-26). To detect the impact of HMGB1 on Dox-induced oxidative stress, DCFH-DA probes were used to assess $\mathrm{H}_{2} \mathrm{O}_{2}$ levels. $\mathrm{H}_{2} \mathrm{O}_{2}$ generation was greatly increased by Dox in the NB cells (Fig. 4A). Importantly, HMGB1-overexpressing NB cells were less sensitive to Dox, evidenced by a decrease in $\mathrm{H}_{2} \mathrm{O}_{2}$ generation following Dox treatment (Fig. 4A). Furthermore, HMGB1-inhibited oxidative stress was partly reversed by the application of 3-MA or Beclin-1 siRNA (Fig. 4B), but not by Atg5 siRNA. Our data suggest that HMGB1 reduced oxidative stress induced by Dox through regulation of Beclin-1-mediated autophagy.

In addition, cleaved PARP and cleaved caspase-3 facilitate cellular disassembly and serve as markers of cells undergoing apoptosis. Thus, the levels of cleaved PARP and cleaved caspase-3 were assessed by western blotting. Our results showed that HMGB1 reduced cleaved PARP and cleaved caspase-3 induced by Dox treatment. Moreover, both 3-MA and Beclin-1 siRNA effectively reversed the effect of HMGB1. Consistently, these data demonstrated that HMGB1 exerted a protective effect against oxidative stress-mediated apoptosis by regulating autophagy.

\section{Discussion}

Neuroblastoma (NB) is one of the predominant tumors that occurs mainly in children. Although a large number of studies from basic research on oncogenes have been carried out, the prognosis of patients, particularly for these with advanced NB, remains poor. Most patients develop chemoresistance and metastatic dissemination (27). The mechanisms involved in drug resistance have not been clarified. Researchers found that HMGB1 is expressed in brain cells and HMGB1 subcellular localization changes during retinoic acid-induced differentiation of P19 NB cells (28). Moreover, HMGB1 protein is released by NB cells upon different stresses, such as carbon dioxide, hypoxia and low pH (29-31). Therefore, we investigated the role of HMGB1-mediated autophagy in NB. We observed that anticancer agents promoted HMGB1 expression, promoted cytosolic HMGB1 expression and the elevation of autophagic activity, suggesting that cytosolic localization of HMGB1 may correlate with autophagy induction. Consistently, cytosolic translocation of HMGB1 as an origin of autophagy has been demonstrated in other cell types under various cytotoxic stresses $(4,17,18)$. Our results showed that the HMGB1 expression in NB cells was increased, and knockdown of HMGB1 rendered them more sensitive to anticancer agents.

In order to clarify the role of HMGB1 in NB cells, the present study using a gain-of-function experiment that showed that overexpression of HMGB1 significantly promoted the proliferative activity and invasive potential of NB cells, indicating that HMGB1 may play an important role in the development and progression of $\mathrm{NB}$, and may represent a potential therapeutic target for the treatment of cancer. Consistent with previous findings, overexpression of HMGB1 was associated with altered hallmarks of autophagy, including LC3-II/I and p62 and we confirmed that HMGB1 serves as a positive regulator of autophagy and possibly mediates the resistance of anticancer agents. To confirm our hypothesis, the apoptosis in HMGB1-overexpressing cells was induced by Dox in the present study for its common clinical application (32). HMGB1 reduced Dox-induced oxidative stress injury and cell death. However, inhibition of autophagy with 3-MA or knockdown of Beclin-1 reversed the effect of HMGB1 and restored the cytotoxicity of Dox treatment, suggesting that cytosolic HMGB1 caused autophagic activation, which resulted in resistance to Dox. Consistent with our results, Mohan et al showed that combination of LC3 short hairpin RNA plasmid transfection inhibited autophagy and increased apoptosis induced by rapamycin in human malignant NB SK-N-BE2 cells (33). These data suggest that HMGB1 is a pro-survival protein, promoting cancer growth and development in NB cells.

Next, we further investigated the mechanism of HMGB1mediated autophagy involved in NB cell death induced by Dox, since autophagy is able to promote or inhibit apoptosis under different stressors $(19,34,35)$. Autophagy in HMGB1overexpressing cells was inhibited by a pharmacological inhibitor (3-MA), siRNA Beclin-1 or siRNA Atg5, respectively, and then the apoptosis and oxidative stress were assessed after Dox treatment. As expected, apoptosis was increased in the HMGB1-overexpressing cells treated with 3-MA or siRNA Beclin-1 exposed to Dox, which is consistent with previous research in several tumor cell lines (36-38), that found decreased protein expression of Beclin-1. However, knockdown of Atg5 failed to reverse the protective effect of HMGB1 against Dox treatment. These data suggest that HMGB1 exerts a protective effect against oxidative stress-mediated apoptosis by regulating Beclin-1-mediated autophagy.

To the best of our knowledge, this is the first study to investigate the role and clinical significance of HMGB1-mediated autophagy in NB. Yet, the use of NB cells from only one cell line provides limited evidence. Further research using more cell lines, xenograft models and primary tumors in vivo are warranted to confirm our hypothesis. In conclusion, our investigation revealed that HMGB1 promotes proliferative activity, invasion and metastasis. Moreover, HMGB1-mediated autophagy exerts a protective effect against oxidative stress-mediated apoptosis by regulating Beclin-1-mediated autophagy.

\section{References}

1. Biedler JL: Drug resistance: Genotype versus phenotype - thirty-second G. H. A. Clowes Memorial Award Lecture. Cancer Res 54: 666-678, 1994.

2. Michaelis M, Klassert D, Barth S, Suhan T, Breitling R, Mayer B, Hinsch N, Doerr HW, Cinatl J and Cinatl J Jr: Chemoresistance acquisition induces a global shift of expression of aniogenesis-associated genes and increased pro-angogenic activity in neuroblastoma cells. Mol Cancer 8: 80, 2009

3. Furfaro AL, Piras S, Passalacqua M, Domenicotti C, Parodi A, Fenoglio D, Pronzato MA, Marinari UM, Moretta L, Traverso N, et al: HO-1 up-regulation: A key point in high-risk neuroblastoma resistance to bortezomib. Biochim Biophys Acta 1842: 613-622, 2014. 
4. Zhang Y, Cheng Y, Ren X, Zhang L, Yap KL, Wu H, Patel R, Liu D, Qin ZH, Shih IM, et al: NAC1 modulates sensitivity of ovarian cancer cells to cisplatin by altering the HMGB1-mediated autophagic response. Oncogene 31: 1055-1064, 2012.

5. Yang S, Xu L, Yang T and Wang F: High-mobility group box-1 and its role in angiogenesis. J Leukoc Biol 95: 563-574, 2014

6. Tang D, Kang R, Cheh CW, Livesey KM, Liang X, Schapiro NE, Benschop R, Sparvero LJ, Amoscato AA, Tracey KJ, et al: HMGB1 release and redox regulates autophagy and apoptosis in cancer cells. Oncogene 29: 5299-5310, 2010.

7. Zhang L, Han J, Wu H, Liang X, Zhang J, Li J, Xie L, Xie Y, Sheng $X$ and $Y u$ J: The association of HMGB1 expression with clinicopathological significance and prognosis in hepatocellular carcinoma: A meta-analysis and literature review. PLoS One 9: e110626, 2014.

8. Süren D, Yıldırım M, Demirpençe Ö, Kaya V, Alikanoğlu AS, Bülbüller N, Yıldız M and Sezer C: The role of high mobility group box 1 (HMGB1) in colorectal cancer. Med Sci Monit 20 : 530-537, 2014

9. Meyer A, Staratschek-Jox A, Springwald A, Wenk H, Wolf J, Wickenhauser C and Bullerdiek J: Non-Hodgkin lymphoma expressing high levels of the danger-signalling protein HMGB1. Leuk Lymphoma 49: 1184-1189, 2008.

10. Brezniceanu ML, Völp K, Bösser S, Solbach C, Lichter P, Joos $S$ and Zörnig M: HMGB1 inhibits cell death in yeast and mammalian cells and is abundantly expressed in human breast carcinoma. FASEB J 17: 1295-1297, 2003.

11. Wu D, Ding Y, Wang S, Zhang Q and Liu L: Increased expression of high mobility group box 1 (HMGB1) is associated with progression and poor prognosis in human nasopharyngeal carcinoma. J Pathol 216: 167-175, 2008.

12. Ueda M, Takahashi Y, Shinden Y, Sakimura S, Hirata H, Uchi R, Takano Y, Kurashige J, Iguchi T, Eguchi H, et al: Prognostic significance of high mobility group box 1 (HMGB1) expression in patients with colorectal cancer. Anticancer Res 34: 5357-5362, 2014.

13. Chen J, Liu X, Zhang J and Zhao Y: Targeting HMGB1 inhibits ovarian cancer growth and metastasis by lentivirus-mediated RNA interference. J Cell Physiol 227: 3629-3638, 2012.

14. Fu LL, Cheng Y and Liu B: Beclin-1: Autophagic regulator and therapeutic target in cancer. Int J Biochem Cell Biol 45: 921-924, 2013.

15. Tang D, Kang R, Livesey KM, Cheh CW, Farkas A, Loughran P, Hoppe G, Bianchi ME, Tracey KJ, Zeh HJ III, et al: Endogenous HMGB1 regulates autophagy. J Cell Biol 190: 881-892, 2010

16. Tang D, Kang R, Livesey KM, Kroemer G, Billiar TR, Van Houten B, Zeh HJ III and Lotze MT: High-mobility group box 1 is essential for mitochondrial quality control. Cell Metab 13: 701-711, 2011.

17. Huang J, Ni J, Liu K, Yu Y, Xie M, Kang R, Vernon P, Cao L and Tang D: HMGB1 promotes drug resistance in osteosarcoma. Cancer Res 72: 230-238, 2012.

18. Pan B, Chen D, Huang J, Wang R, Feng B, Song H and Chen L: HMGB1-mediated autophagy promotes docetaxel resistance in human lung adenocarcinoma. Mol Cancer 13: 165, 2014.

19. Yang YH, Chen K, Li B, Chen JW, Zheng XF, Wang YR, Jiang SD and Jiang LS: Estradiol inhibits osteoblast apoptosis via promotion of autophagy through the ER-ERK-mTOR pathway. Apoptosis 18: 1363-1375, 2013.

20. Wang L, Zhang H, Qian J, Wang K and Zhu J: Interleukin-10 blocks in vitro replication of human cytomegalovirus by inhibiting the virus-induced autophagy in MRC5 cells. Biochem Biophys Res Commun 448: 448-453, 2014.

21. Yang YH, Li B, Zheng XF, Chen JW, Chen K, Jiang SD and Jiang LS: Oxidative damage to osteoblasts can be alleviated by early autophagy through the endoplasmic reticulum stress pathway - implications for the treatment of osteoporosis. Free Radic Biol Med 77: 10-20, 2014.

22. Murai T, Nakagawa Y, Maeda $\mathrm{H}$ and Terada K: Altered regulation of cell cycle machinery involved in interleukin-1-induced $\mathrm{G}_{\mathrm{H}}$ and $\mathrm{G}_{2}$ phase growth arrest of A375S2 human melanoma cells. J Biol Chem 276: 6797-6806, 2001.
23. Petiot A, Ogier-Denis E, Blommaart EF, Meijer AJ and Codogno P: Distinct classes of phosphatidylinositol 3'-kinases are involved in signaling pathways that control macroautophagy in HT-29 cells. J Biol Chem 275: 992-998, 2000.

24. Moreira AC, Branco AF, Sampaio SF, Cunha-Oliveira T, Martins TR, Holy J, Oliveira PJ and Sardão VA: Mitochondrial apoptosis-inducing factor is involved in doxorubicin-induced toxicity on $\mathrm{H} 9 \mathrm{c} 2$ cardiomyoblasts. Biochim Biophys Acta 1842: 2468-2478, 2014

25. Singla S, Kumar NR and Kaur J: In vivo studies on the protective effect of propolis on doxorubicin-induced toxicity in liver of male rats. Toxicol Int 21: 191-195, 2014.

26. Shokoohinia Y, Hosseinzadeh L, Moieni-Arya M, Mostafaie A and Mohammadi-Motlagh HR: Osthole attenuates doxorubicin-induced apoptosis in PC12 cells through inhibition of mitochondrial dysfunction and ROS production. Biomed Res Int 2014: 156848, 2014.

27. Tonini GP and Pistoia V: Molecularly guided therapy of neuroblastoma: A review of different approaches. Curr Pharm Des 12: 2303-2317, 2006

28. Guazzi S, Strangio A, Franzi AT and Bianchi ME: HMGB1, an architectural chromatin protein and extracellular signalling factor, has a spatially and temporally restricted expression pattern in mouse brain. Gene Expr Patterns 3: 29-33, 2003.

29. Faraco G, Fossati S, Bianchi ME, Patrone M, Pedrazzi M, Sparatore B, Moroni F and Chiarugi A: High mobility group box 1 protein is released by neural cells upon different stresses and worsens ischemic neurodegeneration in vitro and in vivo. J Neurochem 103: 590-603, 2007.

30. Reismann M, Wehrmann F, Schukfeh N, Kuebler JF, Ure B and Glüer S: Carbon dioxide, hypoxia and low $\mathrm{pH}$ lead to overexpression of $c-m y c$ and $H M G B-1$ oncogenes in neuroblastoma cells. Eur J Pediatr Surg 19: 224-227, 2009.

31. Pedrazzi M, Averna M, Sparatore B, Patrone M, Salamino F, Marcoli M, Maura G, Cervetto C, Frattaroli D, Pontremoli S, et al: Potentiation of NMDA receptor-dependent cell responses by extracellular high mobility group box 1 protein. PLoS One 7: e44518, 2012

32. Wittig JC, Bickels J, Priebat D, Jelinek J, Kellar-Graney K, Shmookler B and Malawer MM: Osteosarcoma: A multidisciplinary approach to diagnosis and treatment. Am Fam Physician 65: 1123-1132, 2002.

33. Mohan N, Chakrabarti M, Banik NL and Ray SK: Combination of LC3 shRNA plasmid transfection and genistein treatment inhibited autophagy and increased apoptosis in malignant neuroblastoma in cell culture and animal models. PLoS One 8: e78958, 2013.

34. Degenhardt K, Mathew R, Beaudoin B, Bray K, Anderson D, Chen G, Mukherjee C, Shi Y, Gélinas C, Fan Y, et al: Autophagy promotes tumor cell survival and restricts necrosis, inflammation, and tumorigenesis. Cancer Cell 10: 51-64, 2006.

35. Lockshin RA and Zakeri Z: Apoptosis, autophagy, and more. Int J Biochem Cell Biol 36: 2405-2419, 2004.

36. Zhao Z, Tao L, Shen C, Liu B, Yang Z and Tao H: Silencing of Barkor/ATG14 sensitizes osteosarcoma cells to cisplatin-induced apoptosis. Int J Mol Med 33: 271-276, 2014.

37. Lanvers-Kaminsky C, Winter B, Koling S, Frodermann B, Braun Y, Schaefer KL, Diallo R, Koenemann S, Wai D, Willich N, et al: Doxorubicin modulates telomerase activity in Ewing's sarcoma in vitro and in vivo. Oncol Rep 14: 751-758, 2005.

38. Zhou Y, Sun K, Ma Y, Yang H, Zhang Y, Kong X and Wei L: Autophagy inhibits chemotherapy-induced apoptosis through downregulating Bad and Bim in hepatocellular carcinoma cells. Sci Rep 4: 5382, 2014. 